\title{
Caregiver-Reported Quality Measures and Their Correlates in Home Hospice Care
}

Veerawat Phongtankuel, MD, MS, ${ }^{1 * *}$ M.C. Reid, MD, PhD, ${ }^{1}$ Sara J. Czaja, PhD, ${ }^{1}$ Jeanne Teresi, PhD, ${ }^{2,3}$

Joseph P. Eimicke, MS, ${ }^{2}$ Jian X. Kong, MS, ${ }^{2}$ Holly Prigerson, PhD, ${ }^{1}$ Ariel Shalev, BBA, ${ }^{1}$ Ritchell Dignam, MD, ${ }^{4}$

Rosemary Baughn, RN, MSN, ${ }^{4}$ and Ronald D. Adelman, $\mathrm{MD}^{1}$

\begin{abstract}
Background: A majority of hospice care is delivered at home, with significant caregiver involvement. Identifying factors associated with caregiver-reported quality measures could help improve hospice care in the United States. Objectives: To identify correlates of caregiver-reported quality measures: burden, satisfaction, and quality of end-of-life (EoL) care in home hospice care.

Design: A cross-sectional study was conducted from April 2017 through February 2018.

Setting/Subjects: A nonprofit, urban hospice organization. We recruited caregivers whose patients were discharged from home hospice care. Eligible caregiver participants had to be 18 years or older, English-speaking, and listed as a primary caregiver at the time the patient was admitted to hospice.

Measures: The (1) short version of the Burden Scale for Family Caregivers; (2) Family Satisfaction with Care; and (3) Caregiver Evaluation of the Quality of End-Of-Life Care.

Results: Caregivers $(n=391)$ had a mean age of 59 years and most were female $(n=297,76.0 \%)$, children of the patient $(n=233,59.7 \%)$, and non-Hispanic White $(n=180,46.0 \%)$. The mean age of home hospice patients was 83 years; a majority had a non-cancer diagnosis $(n=235,60.1 \%)$, were female $(n=250,63.9 \%)$, and were nonHispanic White $(n=210,53.7 \%)$. Higher symptom scores were significantly associated with greater caregiver burden and lower satisfaction with care; but not lower quality of EoL care. Caregivers who were less comfortable managing patient symptoms during the last week on hospice had higher caregiver burden, lower caregiver satisfaction, and lower ratings of quality of EoL care.

Conclusion: Potentially modifiable symptom-related variables were correlated with caregiver-reported quality measures. Our study reinforces the important relationship between the perceived suffering/symptoms of patients and caregivers' hospice experiences.
\end{abstract}

Keywords: caregiving; end of life; hospice; palliative care

\section{Introduction}

Hospice care has become an integral part of care in the United States for many older adults and their families at the end of life (EoL), with more than $48 \%$ of all Medicare decedents receiving hospice services in 2017. ${ }^{1}$ When hospice is delivered at home, caregivers (the vast majority of whom are family members) play a critically important role in the patient's care. They help manage patients' symptoms and comorbid conditions, provide emotional support, and assist with day-to-day caregiving duties. ${ }^{2}$ As a result, caregivers are an integral member of the

${ }^{1}$ Division of Geriatrics and Palliative Medicine, Weill Cornell Medicine, New York, New York, USA.

${ }^{2}$ Research Division, Hebrew Home at Riverdale, New York, New York, USA.

${ }^{3}$ Columbia University Stroud Center at New York State Psychiatric Institute, New York, New York, USA.

${ }^{4}$ Visiting Nurse Service of New York, New York, New York, USA.

*Address correspondence to: Veerawat Phongtankuel, MD, MS, Weill Cornell Medicine, 525 East 68th Street, Box 39, New York, NY 10065, USA, E-mail: vep9012@med.cornell.edu

(c) Veerawat Phongtankuel et al., 2020; Published by Mary Ann Liebert, Inc. This Open Access article is distributed under the terms of the Creative Commons License (http://creativecommons.org/licenses/by/4.0), which permits unrestricted use, distribution, and reproduction in any medium, provided the original work is properly cited. 
patient's care team and a key source of information for assessing the quality of hospice care.

Although there are various ways to measure quality of EoL care, no consensus indicators have been agreed on. ${ }^{3}$ Caregiver-reported quality measures such as perceived burden, satisfaction, and quality of EoL care are aspects of care that are important and commonly discussed in the literature, but they have not been thoroughly explored in the context of home hospice care. ${ }^{4,5}$ Further, research examining caregiver-reported quality measures and their correlates in the home hospice setting is limited. In one study conducted with 44 caregivers receiving hospice care, researchers found that caregivers who were married or were taking care of a patient with end-stage renal disease reported lower family satisfaction with care scores. ${ }^{6}$

Another, more recent study analyzed responses from the Consumer Assessment of Healthcare Providers and Systems (CAHPS) survey, a nationwide hospice quality measure initiated by the Centers for Medicare and Medicaid Services (CMS) and reported by caregivers. The investigators found that type of payer for hospice, caregiver education, and language spoken at home were most predictive of CAHPS survey scores. ${ }^{7}$

To our knowledge, no study has examined correlates of caregiver-reported quality measures such as caregiver burden (short version of the Burden Scale for Family Caregivers [BSFC-s]), caregiver satisfaction (Family Satisfaction with Care [FAMCARE-2]), and quality of EoL care (Caregiver Evaluation of the Quality of End-Of-Life Care [CEQUEL]) in this setting. Understanding correlates of these three measures could provide insight into factors that place caregivers at risk for a poor/difficult hospice experience and suggest approaches to improve quality of care.

The primary objective of our study was to identify correlates of three caregiver-reported quality measures: caregiver burden, quality of EoL care, and caregiver satisfaction in a large urban home hospice program. Given that comfort is a principal goal of hospice care, we hypothesized that higher patient symptom burden (i.e., higher Edmonton Symptom Assessment Scale [ESAS] scores), as reported by the caregiver, would be independently associated with higher caregiver burden, lower satisfaction with care, and lower quality of EoL care after adjusting for relevant covariates.

\section{Methods}

Study design and setting

This cross-sectional study collected data on caregiverreported quality measures in the home hospice setting.
The Institutional Review Boards (IRBs) of Weill Cornell Medicine and the Visiting Nurse Service of New York approved the study.

The Visiting Nurse Service of New York Hospice and Palliative Care (VNSNY-HPC) organization is a nonprofit hospice that serves more than 1000 patients daily and delivers hospice care to patients in the New York City area. In addition to providing home visits by an interdisciplinary team of physicians, nurses, social workers, and spiritual care counselors, VNSNY-HPC provides enrolled patients a medication kit for pain and symptom management and educational materials that describe available support services. A hospice on-call team provides round-the-clock phone service whereby a hospice nurse, nurse practitioner, or physician may be dispatched to the home based on the needs of the patient and family.

\section{Sample assembly}

During the study period (April 2017 through February 2018), VNSNY-HPC staff generated a weekly list that contained the names of all patients discharged from home hospice in the preceding week. Additional information provided by the hospice agency included patient demographic data (age, gender, race/ethnicity, hospice diagnosis-cancer vs. non-cancer, length of stay, reason for discharge), a home hospice utilization variable (use of continuous home hospice care during the last week on hospice), as well as caregiver contact information (name, address, phone number).

Caregivers received a mailed letter introducing the study and informing them to expect a call in two weeks from a member of the research team. Eligible caregiver participants had to be 18 years or older, English speaking, and listed as a primary caregiver (e.g., family or friend) at the time the patient was admitted to the VNSNY-HPC service. A trained research assistant called potential participants, described the study, and obtained verbal consent from interested and eligible individuals. Of the 1848 caregivers contacted, 804 (44\%) did not answer the phone after three attempts to contact them, 653 (35\%) declined participation, and $391(21 \%)$ completed the phone survey interview.

\section{Data collection}

A semistructured interview guide was used to guide the phone interview after consent was obtained. Caregivers received a $\$ 25$ gift card for their participation.

\section{Dependent variables: Quality measures}

Three quality measures were administered to caregivers during the phone interview. Caregiver burden was 
measured by using the BSFC-s. ${ }^{8}$ The internal consistency (ordinal alpha) estimate for this sample was 0.891. Caregivers' appraisals of their satisfaction with care and the quality of EoL care were assessed by using the FAMCARE- ${ }^{9}$ scale and CEQUEL scale, ${ }^{10}$ respectively. The FAMCARE-2 is a 17 -item scale that measures the degree to which family members are satisfied with the health care received by both the patient and the family with respect to information giving, availability of care, psychological care, and physical patient care. This measure has been widely used in palliative care research, specifically in the palliative care setting. ${ }^{9,11}$ The CEQUEL scale is a 13-item instrument that includes unique markers on perceived suffering and prolongation of death. Lower CEQUEL scores are associated with poor bereavement outcomes. ${ }^{12}$ The internal consistency (ordinal alpha) estimate for this sample was 0.826 . Because of missing data in the outcome variables, the analytic sample sizes were less than the total sample, and they varied across outcomes.

\section{Patient covariates}

Patient-level data included age, gender, race/ethnicity, hospice diagnosis (cancer vs. non-cancer), reason for discharge, use of continuous home hospice care during the last week on hospice, and length of hospice stay. Caregivers served as proxy respondents to measure patient symptom prevalence and level of severity. Symptoms were assessed by using the ESAS. Caregivers were asked to recall whether the patient experienced any of the nine symptoms included in the ESAS (i.e., pain, shortness of breath, nausea, tiredness, drowsiness, lack of appetite, depression, anxiety, well-being) and to rate their intensity on a 0 -to-10 scale during the patient's last week on hospice. The ESAS has evidence of good psychometric properties, ${ }^{13}$ and it been used in numerous studies of patients with terminal illnesses and those at the EoL. ${ }^{14-16}$ Although obtaining assessments from patients would be the gold standard, given that most patients were entering the last stage of dying, this was not feasible. However, there is established evidence for the validity of using proxy respondents to assess patient symptoms at the EoL. ${ }^{17-19}$

\section{Caregiver covariates}

The following caregiver demographic data were collected during the telephone interviews: age, gender, race/ethnicity, relationship with the patient, education level, and average hours of caregiving provided per day during the last seven days on home hospice. We measured the care- giver's comfort in managing patients' symptoms by asking participants, "How would you rate your level of comfort managing (patient's name) symptoms during the last week on home hospice care?" Choices ranged from 1 (very comfortable) to 5 (very uncomfortable).

All data obtained from the medical record and through survey questions were entered into Research Electronic Data Capture (REDCap), a secure web application for building and managing databases.

\section{Statistical approach}

Bivariate analyses were conducted to examine patient and caregiver characteristics associated with each individual quality measure. Point-biserial correlation coefficients were used for binary variables and Pearson correlation coefficients were employed for ordinal and continuous variables.

Variables included in multivariable regression analysis were based on clinical importance and also statistical significance in bivariate analysis. Analyses were conducted to evaluate the unique association between the BSFC-s, CEQUEL, FAMCARE-2, and covariates. The pre-specified alpha level was set at 0.05 for each of the outcome variables. Independent regression models were performed, because the correlations of the three outcomes were relatively low (0.07-0.37). Collinearity diagnostics were examined, and sensitivity analyses were conducted removing potentially collinear variables.

Bivariate analyses were performed by using IBM SPSS Statistics version 25 (IBM Corp, 2016), and multivariable analyses were performed by using SAS (SAS Institute, Inc., SAS Version 9.4. Cary, NC: SAS Institute, Inc.; 2015).

\section{Results}

Demographic data for patients and caregivers are presented in Tables 1 and 2. The mean age of patients was 83 years; a majority had a non-cancer diagnosis $(n=235,60.1 \%)$, were female $(n=250,63.9 \%)$, and non-Hispanic White $(n=210,53.7 \%)$. The average length of stay in hospice was 98 days with a median of 33 days. Caregivers had a mean age of 59 years and most were female $(n=297,76.0 \%)$, children of the patient ( $n=233,59.7 \%)$, and had a college education or greater $(n=271,76.1 \%)$. Caregivers reported providing an average of 14 hours of patient care per day during the patient's last week on hospice. Death was the major reason for discharge $(n=351,89.8 \%)$, followed by hospitalization $(n=24,6.1 \%)$, and finally others $(n=16,4.1 \%)$. 
Table 4. Bivariate Analysis of Quality Measures in Home Hospice Population

\begin{tabular}{|c|c|c|c|}
\hline & $\begin{array}{c}\text { BSFC-s } \\
(n=359)\end{array}$ & $\begin{array}{l}\text { FAMCARE-2 } \\
(n=350)\end{array}$ & $\begin{array}{l}\text { CEQUEL } \\
(n=332)\end{array}$ \\
\hline & $r$ & $r$ & $r$ \\
\hline Patient age & -0.075 & -0.077 & -0.013 \\
\hline $\begin{array}{l}\text { Patient gender } \\
\text { Male } \\
\text { Female }\end{array}$ & -0.016 & -0.079 & 0.085 \\
\hline $\begin{array}{l}\text { Patient race/ethnicity } \\
\text { White } \\
\text { Black } \\
\text { Hispanic } \\
\text { Asian } \\
\text { Other/undisclosed }\end{array}$ & $\begin{array}{r}0.022 \\
-0.008 \\
0.035 \\
-0.079\end{array}$ & $\begin{array}{r}-0.014 \\
0.015 \\
-0.030 \\
0.048\end{array}$ & $\begin{array}{r}-0.027 \\
0.017 \\
0.019 \\
-0.003\end{array}$ \\
\hline $\begin{array}{l}\text { Hospice diagnosis } \\
\text { Cancer } \\
\text { Non-cancer }\end{array}$ & 0.012 & -0.101 & -0.039 \\
\hline Length of stay (days) & 0.002 & -0.073 & $-0.107^{*}$ \\
\hline $\begin{array}{l}\text { Reason for discharge from hosp } \\
\text { Death } \\
\text { Hospitalization } \\
\text { Other }^{\mathrm{b}}\end{array}$ & $\begin{array}{l}\mathrm{ce}^{\mathrm{a}} \\
-0.196^{* * *} \\
0.150^{* *}\end{array}$ & $\begin{array}{r}-0.168^{* * *} \\
0.192^{* * * *}\end{array}$ & $\begin{array}{r}0.095 \\
-0.046\end{array}$ \\
\hline $\begin{array}{l}\text { Received continuous home } \\
\text { care during the last week } \\
\text { on hospice } \\
\text { Caregiver age }\end{array}$ & -0.071 & -0.037 & -0.031 \\
\hline $\begin{array}{l}\text { Caregiver gender } \\
\text { Male } \\
\text { Female }\end{array}$ & 0.101 & -0.015 & 0.057 \\
\hline $\begin{array}{l}\text { Caregiver race/ethnicity } \\
\text { White } \\
\text { Black } \\
\text { Hispanic } \\
\text { Asian } \\
\text { Other }^{\mathrm{b}} \\
\text { Not specified }\end{array}$ & $\begin{array}{r}-0.003 \\
-0.038 \\
0.056 \\
-0.074 \\
\\
0.073\end{array}$ & $\begin{array}{r}-0.007 \\
-0.060 \\
-0.030 \\
0.037\end{array}$ & $\begin{array}{r}-0.015 \\
0.044 \\
0.040 \\
0.001 \\
-0.110^{*}\end{array}$ \\
\hline $\begin{array}{l}\text { Caregiver relationship with pati } \\
\text { Child } \\
\text { Spouse } \\
\text { Relative } \\
\text { Grandchild }^{\mathrm{b}} \\
\text { Friend }^{\mathrm{b}} \\
\text { Parent }^{\mathrm{b}}\end{array}$ & $\begin{array}{l}\mathrm{nt}^{\mathrm{a}} \\
0.100 \\
-0.020 \\
-0.118^{*}\end{array}$ & $\begin{array}{r}-0.031 \\
0.060 \\
-0.051\end{array}$ & $\begin{array}{l}0.139^{* *} \\
-0.089 \\
-0.083\end{array}$ \\
\hline Caregiver education level $^{\mathrm{a}}$ & & & \\
\hline $\begin{array}{l}\text { High school } \\
\text { College }\end{array}$ & $\begin{array}{r}-0.050 \\
0.028\end{array}$ & $\begin{array}{c}-0.143^{* *} \\
0.027\end{array}$ & $\begin{array}{r}0.053 \\
-0.009\end{array}$ \\
\hline Graduate school & 0.025 & 0.102 & -0.045 \\
\hline $\begin{array}{l}\text { Average hours per day spent } \\
\text { caregiving during the last } \\
\text { week on hospice }\end{array}$ & 0.022 & 0.023 & -0.017 \\
\hline ESAS & $0.288^{* * *}$ & $0.200^{* * * *}$ & $-0.110^{*}$ \\
\hline Comfort managing symptoms ${ }^{c}$ & $0.176^{* * *}$ & $0.359^{* * * *}$ & $-0.192^{* * *}$ \\
\hline
\end{tabular}

BSFC-s: Higher scores are associated with greater caregiver burden. FAMCARE-2: Higher scores are associated with lower satisfaction. CEQUEL: Lower scores are associated with poor bereavement.

${ }^{\mathrm{a}}$ Dummy variables used as reference group.

${ }^{\mathrm{b}}$ Not computed due to sparse data.

'Scale from 1 to 5 with $1=$ very comfortable and $5=$ very uncomfortable.

${ }^{*} p \leq 0.05,{ }^{* *} p \leq 0.01,{ }^{* * *} p \leq 0.001$

ESAS, Edmonton Symptom Assessment Scale.
Quality of EoL care (CEQUEL) scores

and correlates

The mean CEQUEL score (Table 3) was 22.4 $(\mathrm{SD}=3.0)$. Table 4 shows bivariate correlation coefficients and $p$-values for CEQUEL score and patient, caregiver, and hospice utilization variables. Lower quality of EoL care scores were associated with longer length of stay $(r=-0.107, p \leq 0.05)$, caregivers who did not specify their race $(r=-0.110, p \leq 0.05)$, caregivers who were children of the patient $(r=0.139$, $p \leq 0.05)$, higher ESAS score $(r=-0.110, p \leq 0.05)$, and caregivers who were less comfortable managing patient symptoms $(r=-0.192, p \leq 0.01)$.

Table 5 shows the results of the linear regression model predicting CEQUEL score. Lower ratings of quality of EoL care were associated with longer hospice length of stays (estimate $=-0.002, p=0.022$ ) and caregivers who were less comfortable managing patient symptoms (estimate $=-0.322, p=0.012$ ). Contrary to our primary study hypothesis, CEQUEL scores were not significantly related to symptom burden at the multivariate level.

\section{Discussion}

Our study examined the correlates of caregiverreported quality measures in home hospice care. We found that caregiver comfort in managing patient symptoms during the last week on hospice was associated with all three quality measures examined: caregiver burden, caregiver satisfaction, and quality of EoL care. We also found that both higher caregiverreported symptom scores and caring for patients who did not die in hospice were associated with higher caregiver burden and lower satisfaction with care.

We used the BSFC-s to measure caregiver burden in this study. The mean score for our sample was 15.5 $(\mathrm{SD}=5.5)$. To provide some context, a study of caregivers of dementia patients in Germany found a lower mean BSFC-s score of $10.2(\mathrm{SD}=8.0) .{ }^{8}$ Overall satisfaction was high in our sample, which is consistent with many studies examining satisfaction with hospice care. ${ }^{20}$ Lastly, our reported quality of EoL care scores $($ mean $=22.4, \mathrm{SD}=3.0)$ are similar to a previous study conducted by Higgins and Prigerson looking at CEQUEL scores in advanced cancer patients and their caregivers $($ mean $=23.6, \mathrm{SD}=2.2) .{ }^{10}$

We hypothesized that higher caregiver-reported patient symptom scores (i.e., ESAS) would be associated with all three outcomes based on our clinical experience caring for this population and past work linking 
Table 5. Linear Regression Analysis for Quality Measures in Home Hospice Population

\begin{tabular}{|c|c|c|c|c|c|c|c|c|c|}
\hline & \multicolumn{3}{|c|}{ BSFC-s $(n=359)$} & \multicolumn{3}{|c|}{ FAMCARE-2 $(n=350)$} & \multicolumn{3}{|c|}{ CEQUEL scale $(n=332)$} \\
\hline & Estimate & SE & $p$ & Estimate & SE & $p$ & Estimate & SE & $p$ \\
\hline Intercept & 15.127 & 3.039 & $<0.001$ & 18.980 & 7.157 & 0.008 & 23.567 & 1.893 & $<0.001$ \\
\hline ESAS (higher score indicates greater distress) & 0.074 & 0.017 & $<0.001$ & 0.084 & 0.039 & 0.034 & -0.017 & 0.010 & 0.103 \\
\hline Patient's age & 0.014 & 0.030 & 0.643 & 0.084 & 0.070 & 0.231 & -0.013 & 0.018 & 0.459 \\
\hline Patient female & 0.472 & 0.608 & 0.438 & -1.331 & 1.416 & 0.348 & 0.506 & 0.362 & 0.163 \\
\hline Patient white & 0.421 & 0.599 & 0.482 & -2.091 & 1.398 & 0.136 & -0.076 & 0.355 & 0.831 \\
\hline Cancer hospice diagnosis & -1.084 & 0.657 & 0.100 & 1.765 & 1.536 & 0.252 & 0.275 & 0.395 & 0.488 \\
\hline Length of stay & 0.001 & 0.002 & 0.877 & -0.004 & 0.004 & 0.312 & -0.002 & 0.001 & 0.022 \\
\hline Death discharge reason & -3.288 & 0.951 & 0.001 & -5.191 & 2.199 & 0.019 & 0.844 & 0.697 & 0.227 \\
\hline Received continuous home care during last week on hospice & 1.161 & 0.956 & 0.225 & 0.754 & 2.257 & 0.739 & 0.389 & 0.556 & 0.484 \\
\hline Caregiver age & 0.080 & 0.030 & 0.008 & -0.104 & 0.071 & 0.140 & 0.010 & 0.018 & 0.559 \\
\hline Caregiver female & 1.063 & 0.674 & 0.116 & -0.234 & 1.569 & 0.881 & 0.346 & 0.396 & 0.383 \\
\hline Caregiver spouse & 0.844 & 0.990 & 0.395 & 3.027 & 2.319 & 0.193 & -0.825 & 0.588 & 0.162 \\
\hline Caregiver education & 0.367 & 0.391 & 0.349 & 2.187 & 0.912 & 0.017 & -0.064 & 0.229 & 0.782 \\
\hline Number of hours with patient per day & 0.017 & 0.033 & 0.618 & -0.004 & 0.077 & 0.957 & -0.008 & 0.020 & 0.696 \\
\hline Comfort managing symptoms during last week on hospice care ${ }^{a}$ & 0.413 & 0.210 & 0.050 & 2.987 & 0.490 & $<0.001$ & -0.322 & 0.127 & 0.012 \\
\hline
\end{tabular}

BSFC-s: Higher scores are associated with greater caregiver burden. FAMCARE-2: Higher scores are associated with lower satisfaction. CEQUEL: Lower scores are associated with poor bereavement.

${ }^{a}$ Scale from 1 to 5 with $1=$ very comfortable and $5=$ very uncomfortable.

$\mathrm{SE}$, standard error.

symptoms to poor patient outcomes such as hospitalization. $^{21,22}$ We did find that higher caregiver-reported ESAS scores were associated with two of the quality measures, caregiver burden and satisfaction with care, but not with quality of EoL care. This may be a result of the questions on the CEQUEL survey, which captures aspects of quality other than patient symptom burden such as prolongation of death and shared decision making.

Along the same lines, we did find that caregivers of patients who died on hospice compared with those discharged alive (i.e., hospitalized or discharged for other reasons) had lower caregiver burden scores and higher caregiver satisfaction ratings. Reducing symptoms and avoidable care transitions at the EoL are difficult challenges to address and further research aimed at treating symptoms, supporting caregivers, and finding solutions to reduce unnecessary hospice transitions is needed.

In our regression analysis, caregivers' comfort level managing symptoms during the last week on hospice was independently associated with all three quality measures. This finding is of interest and seems particularly pertinent to home hospice care. Caregivers spend a significant amount of time caring for patients. ${ }^{2}$ We speculate that caregivers who are more comfortable managing symptoms feel they are providing better palliative care, which may lead to better reported quality measures. Further research is needed to validate and measure caregiver efficacy in providing EoL care and understand its longitudinal impact on outcomes.

Based on our analysis, interventions to help improve caregivers' knowledge and skills in understanding and managing symptoms may be appropriate. ${ }^{23,24}$ We have shown in our qualitative work ${ }^{25}$ that caregivers expressed the need for more knowledge around what to expect at the EoL. Past interventions conducted by Cagle et al. ${ }^{23}$ and Campbell and McErlane ${ }^{24}$ to address pain and dyspnea, respectively, can be building blocks to support home hospice caregivers. It will be important for future work to better describe the spectrum of caregiver roles in home hospice care, as well as the social and educational supports they receive, which may vary considerably depending on the underlying diagnosis of the care recipient, family makeup, and hospice organization, to provide more tailored approaches to help support them.

From a clinical standpoint, our study reinforces the important relationship between perceived suffering/ symptoms of patients and caregivers' home hospice experiences. Although we know that hospice improves patients' quality of life ${ }^{26}$ and many caregivers report high satisfaction with care, studies also have found that burdensome symptoms are still prevalent. ${ }^{27,28}$ Hospices should continue to strive to improve how symptoms are both evaluated and managed. In terms of future research, we believe that advancement in detecting and treating signs and symptoms is one area that warrants further study. Further, given the critically important role that caregivers play in this setting, finding ways to support and educate caregivers, whether it is through better access to clinical supervision, support through advancing technologic aides (e.g., telemedicine, online educational videos), or other models of care delivery, are important to understand, develop, and rigorously test. 
Our study has several limitations. First, we interviewed caregivers instead of patients to measure ESAS scores. Although it would have been preferable to obtain ESAS data from patients, we had concerns about recruitment (e.g., being able to obtain patient consent and adequate sample size), along with concerns about the potential burden of administering surveys to patients at the EoL. We, therefore, elected to collect proxy data from caregivers postdischarge. ${ }^{17-19}$ In addition, caregivers' comfort level managing patient symptoms and recall bias may have impacted reporting of symptoms. Second, despite our multiple attempts to reach potential participants, our refusal and nonresponse rates were high, which highlights the recruitment challenges researchers face when conducting EoL/hospice research, ${ }^{29}$ and may have biased the sample. We did find variations in the average length of stay (98 vs. 82 days), proportion of patients who were discharged due to death ( $90 \%$ vs. $86 \%)$, and proportion of patients with a cancer diagnosis (40\% vs. $48 \%$ ) between respondents and non-respondents. Third, although we had a diverse sample in terms of race/ethnicity, a majority of participants were highly educated with either college or graduate school education. Lastly, we only recruited from one nonprofit, urban hospice organization, which may not reflect the national makeup of caregivers and patients receiving home hospice care.

In conclusion, our study showed that caregiver-reported quality measures (i.e., caregiver burden, caregiver satisfaction, and quality of EoL care) were associated with symptom-related variables. Further research and strategies are needed to improve symptom management for patients and support caregivers in this area to improve quality of care in the home hospice setting.

\section{Acknowledgments}

The authors would like to acknowledge and express their deepest gratitude to the caregivers who participated in this study.

\section{Funding Information}

The authors disclosed receipt of the following financial support: V.P. is supported by grants from the National Institute of Aging (R03 AG053284 and K76 AG059997) and Weill Cornell Medical College Clinical and Translational Science Center. M.C.R. is supported by the following grants from the National Institute on Aging: P30AG022845 and K24AGO53462. H.P. is supported by grants from the National Cancer Institute (CA197730 and CA218313) and the National Institute of Nursing Research (NR018693).

\section{Author Disclosure Statement}

No competing financial interests exist.

\section{References}

1. NHPCO Facts and Figures 2018. National Hospice and Palliative Care Organization (online): https://www.nhpco.org/wpcontent/uploads/ 2019/07/2018_NHPCO_Facts_Figures.pdf. (Last accessed November 24, 2019).

2. Ornstein KA, Kelley AS, Bollens-Lund E, Wolff JL: A national profile of end-of-life caregiving in the United States. Health Aff 2017;36:11841192.

3. Dy SM, Kiley KB, Ast K, et al: Measuring what matters: Top-ranked quality indicators for hospice and palliative care from the American Academy of Hospice and Palliative Medicine and Hospice and Palliative Nurses Association. J Pain Symptom Manage 2015;49:773-781.

4. Connor SR, Teno J, Spence C, Smith N: Family evaluation of hospice care: Results from voluntary submission of data via website. J Pain Symptom Manage 2005;30:9-17.

5. Andrews SC: Caregiver burden and symptom distress in people with cancer receiving hospice care. Oncol Nurs Forum 2001;28:14691474.

6. Meyers JL, Gray LN: The relationships between family primary caregiver characteristics and satisfaction with hospice care, quality of life, and burden. Oncol Nurs Forum 2001;28:73-82.

7. Parast L, Haas A, Tolpadi A, et al: Effects of caregiver and decedent characteristics on CAHPS hospice survey scores. J Pain Symptom Manage 2018;56:519.e1-529.e1.

8. Graessel E, Berth H, Lichte T, Grau H: Subjective caregiver burden: Validity of the 10-item short version of the Burden Scale for Family Caregivers BSFC-s. BMC Geriatr 2014;14:23.

9. Aoun S, Bird S, Kristjanson LJ, Currow D: Reliability testing of the FAMCARE-2 scale: Measuring family carer satisfaction with palliative care. Palliat Med 2010;24:674-681.

10. Higgins PC, Prigerson HG: Caregiver evaluation of the quality of end-oflife care (CEQUEL) scale: The caregiver's perception of patient care near death. PLoS One 2013;8:e66066.

11. Naoki Y, Matsuda Y, Maeda I, et al: Association between family satisfaction and caregiver burden in cancer patients receiving outreach palliative care at home. Palliat Support Care 2018;16:260-268.

12. Higgins PC, Garrido MM, Prigerson HG: Factors predicting bereaved caregiver perception of quality of care in the final week of life: Implications for health care providers. J Palliat Med 2015;18:849-857.

13. Chang VT, Hwang SS, Feuerman M: Validation of the edmonton symptom assessment scale. Cancer 2000;88:2164-2171.

14. Chow E, Wong R, Connolly R, et al: Prospective assessment of symptom palliation for patients attending a rapid response radiotherapy program: Feasibility of telephone follow-up. J Pain Symptom Manage 2001;22:649656.

15. De La Cruz M, Noguera A, San Miguel-Arregui MT, et al: Delirium, agitation, and symptom distress within the final seven days of life among cancer patients receiving hospice care. Palliat Support Care 2015;13:211216.

16. Gilbertson-White $S$, Aouizerat $B E$, Jahan $T$, Miaskowski $C$ : A review of the literature on multiple symptoms, their predictors, and associated outcomes in patients with advanced cancer. Palliat Support Care 2011;9:81102.

17. McPherson CJ, Wilson KG, Lobchuk MM, Brajtman S: Family caregivers' assessment of symptoms in patients with advanced cancer: Concordance with patients and factors affecting accuracy. J Pain Symptom Manage 2008;35:70-82.

18. Lobchuk MM: The memorial symptom assessment scale: Modified for use in understanding family caregivers' perceptions of cancer patients' symptom experiences. J Pain Symptom Manage 2003;26:644-654.

19. Lobchuk MM, Degner LF: Symptom experiences: Perceptual accuracy between advanced-stage cancer patients and family caregivers in the home care setting. J Clin Oncol 2002;20:3495-3507.

20. Candy B, Holman A, Leurent B, et al: Hospice care delivered at home, in nursing homes and in dedicated hospice facilities: A systematic review of quantitative and qualitative evidence. Int J Nurs Stud 2011; 48:121-133. 
21. Phongtankuel V, Scherban BA, Reid MC, et al: Why do home hospice patients return to the hospital? A study of hospice provider perspectives. J Palliat Med 2016;19:51-56.

22. Phongtankuel V, Paustain S, Reid M, et al: Events leading to hospitalization of home hospice patients: A study of primary caregivers' perspectives. Palliat Med 2016:1-6.

23. Cagle JG, Zimmerman S, Cohen LW, et al: EMPOWER: An intervention to address barriers to pain management in hospice. J Pain Symptom Manage 2015;49:1-12.

24. Campbell ML, McErlane L: Feasibility of a study to test the effectiveness of a dyspnea assessment and treatment bundle to improve family caregiving of patients in home hospice. J Palliat Med 2018;21: 1547.

25. Shalev A, Phongtankuel V, Reid MC, et al: Home hospice caregivers' perceived information needs. Am J Hosp Palliat Med 2018;36:302307.

26. Teno JM, Gozalo PL, Lee IC, et al: Does hospice improve quality of care for persons dying from dementia? J Am Geriatr Soc 2011;59:15311536.

27. Kutner JS, Bryant LL, Beaty BL, Fairclough DL: Time course and characteristics of symptom distress and quality of life at the end of life. J Pain Symptom Manage 2007:34:227-236.

28. McMillan SC, Small BJ: Symptom distress and quality of life in patients with cancer newly admitted to hospice home care. Oncol Nurs Forum 2002;29:1421-1428.

29. Jordhøy MS, Kaasa S, Fayers $\mathrm{P}$, et al: Challenges in palliative care research; recruitment, attrition and compliance: Experience from a randomized controlled trial. Palliat Med 1999;13:299-310.
Cite this article as: Phongtankuel V, Reid MC, Czaja SJ, Teresi J, Eimicke JP, Kong JX, Prigerson H, Shalev A, Dignam R, Baughn R, Adelman RD (2020) Caregiver reported quality measures and their correlates in home hospice care, Palliative Medicine Reports 1:1, 111-118, DOI: 10.1089/pmr.2020.0055.

Abbreviations Used
BSFC-s $=\begin{gathered}\text { short version of the Burden Scale } \\ \text { for Family Caregivers }\end{gathered}$
CAHPS $=\begin{gathered}\text { Consumer Assessment of Healthcare Providers } \\ \text { and Systems }\end{gathered}$
CEQUEL $=$ Caregiver Evaluation of the Quality of End-Of-Life Care
CMS $=$ Centers for Medicare and Medicaid Services
EOL $=$ end of life
ESAS $=$ Edmonton Symptom Assessment Scale.
FAMCARE-2 $=$ Family Satisfaction with Care
IRBs $=$ Institutional Review Boards
REDCap $=$ Research Electronic Data Capture
SD $=$ standard deviation
SE $=$ standard error
VNSNY-HPC $=$ Visiting Nurse Service of New York Hospice
and Palliative Care

\section{Publish in Palliative Medicine Reports}

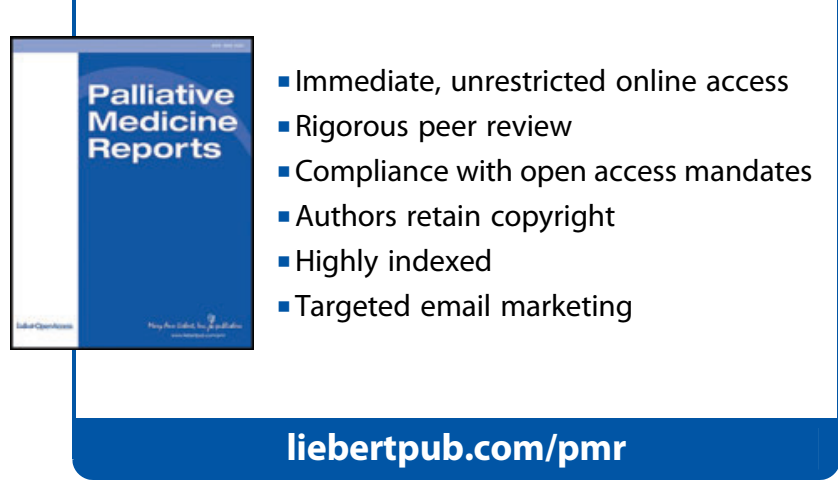

